\title{
The "Creative Dolphin" Revisited: What Do Dolphins Do When Asked to Vary their Behavior?
}

\author{
Stan A. Kuczaj II ${ }^{1 *}$ and Holli C. Eskelinen ${ }^{2}$ \\ ${ }^{1}$ University of Southern Mississippi \\ ${ }^{2}$ Dolphins Plus \\ *Corresponding author (Email: s.kuczaj@usm.edu)
}

Citation - Kuczaj, S. A., II, \& Eskelinen, H. C. (2014). The "creative dolphin" revisited: What do dolphins do when asked to vary their behavior? Animal Behavior and Cognition, 1(1), 66-77. doi: 10.12966/abc.02.05.2014

\begin{abstract}
The variability of dolphin behavior is evident in their communication, foraging, and play. Dolphins can also vary their behavior when asked to do so by humans. Following the work of Karen Pryor and her colleagues, this ability is commonly referred to as creative behavior, a bit of a misnomer since dolphins need not always create a new behavior to succeed on this task. Nonetheless, when given a task in which success depends on not repeating what one has already done, dolphins are able to remember what they have done and successfully produce a new behavior, sometimes even a completely novel one. In this paper, we report similarities and differences in the performance of three bottlenose dolphins (Tursiops truncatus) given such a task, the results highlighting the need for continued investigation of individual differences in cognitive style and performance.
\end{abstract}

Keywords - Innovation, creativity, bottlenose dolphin, Tursiops truncatus, variability, novelty, memory

Dolphins are not all the same. They exhibit both distinct individual personalities and substantial differences between groups (Highfill \& Kuczaj, 2007; Kuczaj, Highfill, \& Byerly, 2012). For example, dolphins within a group tend to produce individually distinctive whistles that seem to signal individual identity, such whistles being acquired during the first year or so of a dolphin's life (Caldwell, Caldwell, \& Tyack, 1990; Janik \& Sayigh, 2013; Kuczaj, in press; Tyack \& Sayigh, 1997). However, there is considerable variability in the age at which a calf first produces signature whistles (Tyack \& Sayigh, 1997). Signature whistles appear in the first weeks of life for some calves, but only occur in the whistle repertoire of other calves that have passed their first birthday. In addition, the extent to which a calf's signature whistle matches that of its mother, as well as the speed with which a calf develops its own signature whistle, seems to depend on the nature of the relationship between the calf and the mother (Tyack \& Sayigh, 1997). A strong mother-calf bond may facilitate the calf's acquisition of its signature whistle. In addition, such a bond may also result in a calf learning a signature whistle that resembles its mother's whistle more so than would the signature whistle of a calf with a less secure attachment to its mother.

Dolphins also differ in terms of the foraging strategies that they use. These include the use of air bubbles to confuse and capture prey (Fertl \& Wilson, 1997), obtaining and placing sponges on their rostrums to protect themselves from injury while scouring the ocean floor (Smolker, Richards, Connor, Mann, \& Berggren, 1997), chasing fish onto sloped muddy beaches or into shallow water and then stranding themselves in order to capture the fish (Duffy-Echevarria, Connor, \& St. Aubin, 2008; Peddemors \& Thompson, 1994; Sargeant, Mann, Berggren, \& Krützen, 2005), creating mud plumes to 
trap and confuse fish (Lewis \& Schroeder, 2003), corkscrewing into the substrate with their rostrums (without sponges) to capture hidden prey (Herzing, 1996; Rossbach \& Herzing, 1998), cooperating to herd prey into large bait balls (Benoit-Bird \& Au, 2009), and even cooperating with human fisherman to enhance the foraging success of both species (Daura-Jorge, Cantor, Ingram, Lusseau, \& Simõn-Lopes, 2012).

Some foraging strategies require groups of animals, others involve only an individual, and some actually concern the preparation of the meal once it is caught. Finn, Tregenza, and Norman (2009) reported that a bottlenose dolphin would herd a cuttlefish to a sand substrate, then pin it to the sea floor and kill it. However, she did not immediately eat the cuttlefish. First, the dolphin moved the cuttlefish from the sea floor and then beat it with her rostrum until all the ink was removed. The dolphin next returned the cuttlefish to the sea floor, where she placed it upside down in the sand and pushed it along the bottom to remove the skin and cuttlebone. This behavior has not been observed in other dolphins, but Finn et al. suspect that at least some other dolphins in this area use similar techniques to improve the quality of their cuttlefish prey.

The suggestion by Finn et al. (2009) that additional dolphins may prepare their cuttlefish prey much as the dolphin they observed is partially based on the reasonable assumption that members of a dolphin community use similar foraging techniques. However, such is not always the case. Only a subset of the dolphins in Shark Bay, Australia use sponges during foraging (Mann et al., 2008). Dolphins that use sponges are more likely to be solitary, spend more time foraging, inhabit deeper water areas, and dive for longer periods than are dolphins in the same area that do not use sponges. Moreover, the cultural transmission of this behavior appears to be limited to a single matriline with all spongers being the descendants of a single female (Krützen et al., 2005).

Similarly, only select dolphins in Shark Bay, Australia engage in beach hunting by chasing fish into shallow water and sometimes temporarily stranding themselves in order to capture the fish (Sargeant et al., 2005). Ten years of observations in this area yielded only four adults and their calves that foraged in this manner, with calves apparently learning beach hunting from their mothers.

Such differences are not limited to Shark Bay dolphins. The dolphins in Laguna, Brazil that cooperate with human beach-casting fisherman comprise a subset of the local population (Daura-Jorge et al., 2012). Individual differences in the foraging behaviors of dolphins also occur in Sarasota Bay, Florida and were summarized by Nowacek (2002). Thus, the foraging plasticity of dolphins is reflected in both differences among animals and in the flexibility demonstrated by individual animals (Nowacek, 2002; Spitz, Richard, Meynier, Pusineri, \& Ridoux, 2006). Given the potential significance of observational learning in the foraging behaviors of dolphins (and in other aspects of dolphin lives, as well), the fact that dolphins choose which dolphins to observe and mimic may influence the variability that occurs in foraging within a community (Kuczaj, Yeater, \& Highfill, 2012).

Dolphins can also learn to vary their behaviors when asked to do so by humans. The first demonstration of this ability was reported by Karen Pryor and her colleagues (Pryor, 1975; Pryor, Haag, \& O'Reilly, 1969). They trained two rough-toothed dolphins (Steno bredanensis) to vary the behaviors the dolphins produced across training sessions by reinforcing behaviors that the dolphins had not performed in previous sessions. In essence, the dolphins had to learn that during these sorts of sessions "only things which have not been previously reinforced are reinforceable" (Pryor, 1975, p. 236).

More recently, dolphins have been asked to produce novel behaviors within as well as across training sessions (Braslau-Schneck, 1994; Herman, 2006). Although such sessions are commonly referred to as "creative" or "innovative" in the dolphin cognition literature, a dolphin need not create a new behavior every time this request is made. Instead, the constraint facing the dolphins is to not repeat an immediately preceding behavior (Mercado, Murray, Uyeyama, Pack, \& Herman, 1998) or to not produce a behavior that was already produced that session. Dolphins are able to learn these constraints and thus perform well on such tasks. In addition, when asked to do so, dolphins can repeat the behavior they were asked to do immediately before the "repeat" request is given (Mercado et al., 1998). This, combined with their ability to produce a behavior that differs from ones they produced earlier, suggests that dolphins 
represent their actions in such a way that they can use these representations to guide subsequent behaviors.

In this paper, we describe the behaviors produced by three Atlantic bottlenose dolphins (Tursiops truncatus) when they were asked to vary their behavior by not repeating behaviors they had previously produced during the test session. We did not require that the animals actually create novel behaviors that they had not produced before (although they sometimes did so). Instead, in order to be successful a dolphin had to produce a behavior that it had not already used that session. Thus, the "vary" signal actually informed the dolphin to not repeat any of the behaviors it had produced the previous times it had seen that signal during that session. Although dolphins did produce novel behaviors in this context, the "vary" task described here is actually more of a memory task than a "creativity" task for two reasons: (1) the dolphin must remember what it has done that session, and (2) dolphins can produce known behaviors as long as the behavior was not used previously that session.

We examined the performance of each of the dolphins in the "vary" task in order to assess: (1) individual differences in the extent to which dolphins can remember the behaviors they recently performed, and (2) the different strategies employed by individual dolphins when asked to modify their behavior.

\section{Method}

\section{Subjects}

Three bottlenose dolphins (Tursiops truncatus) housed at Dolphin Cove, a natural seawater facility, in Key Largo, Florida, were the focus for this study. The dolphins were all males, two of which were approximately 20 years old at the time of this study (Alfonz and Kimbit). The other dolphin, Leo, was approximately 10 years old when he participated in this study. Each dolphin was born at either Dolphin Cove or its sister facility, Dolphins Plus.

\section{Procedure}

Training. Each dolphin was trained using positive reinforcement to respond to a trainer's request to produce a behavior they had not yet performed during that session by being reinforced when they did so. In order to be reinforced, a dolphin could produce a previously learned and reinforced behavior, create a novel behavior, or combine behaviors in a unique way, so long as the behavior or behavior sequence had not been previously used that session. Conceptual training of the "vary" behavior began at the same time for each dolphin. As training of the "vary" behavior progressed, each subject went from producing simplistic behaviors (e.g., stationary whistling) to more complex behaviors with additional components (e.g., waving with pectoral fin while whistling), although the rate and frequency with which each dolphin increased the complexity of its behaviors varied from dolphin to dolphin. During training, dolphins were reinforced for novel sequences of behaviors, but were not reinforced for producing sequences of behaviors that they had already produced. If a dolphin appeared to be learning a routine and so started each new session with the same behaviors (e.g., pectoral fin wave while diving under the surface of the water), the dolphin was not reinforced in order to prevent him from learning to produce set sequences of behaviors in this context.

Training sessions were conducted at least once each week, but the number of sessions each week and the duration of each session varied across and within each dolphin's training. Nonetheless, the number of sessions necessary before a dolphin demonstrated that it understood the task was remarkably consistent across dolphins. The criterion for mastery of the task was four consecutive sessions in which the dolphin performed a minimum of three different behaviors without repeating a behavior. Alfonz and Leo each required 69 sessions to achieve criterion, while Kimbit required 74 sessions. Test trials began once a dolphin demonstrated that it understood the task. The data we report below are only from test sessions that followed a dolphin achieving criterion. 
Test trials. Dolphins were tested individually. Each trial consisted of a trainer presenting a dolphin with the "vary" signal, the dolphin's subsequent response, the trainer presenting the "vary" signal again, the dolphin's response, the trainer presenting the "vary" signal again if the dolphin had produced a different behavior than the first one, the dolphin's response, and so on until the dolphin failed to produce a new behavior or the trainer ended the trial. Alfonz received 54 trials, Kimbit received 48 trials, and Leo received 59 trials.

All trials were recorded on video with GoPro Hero digital cameras. Behaviors were coded using these video recordings. Coding consisted of listing the specific behavior produced following each "vary" signal, and determining if this behavior was a single behavior (e.g., a stationary pectoral fin wave) or a combination of behaviors (e.g., swimming in a circle while waving a pectoral fin). To keep nomenclature straightforward, we use "behavior" to refer to the overall behavior that occurred following the "vary" signal, regardless of how simple or complex the behavior was. "Component" is used to refer to the number of individual behaviors that comprised the overall behavior following the "vary" signal.

\section{Results}

As shown in Figure 1, each of the dolphins was outstanding in terms of varying their behavior when instructed to do so by a trainer. Each dolphin failed to vary its behavior following the first opportunity to do so on three separate trials ( 3 out of 54 trials for Alfonz, 3 out of 48 trials for Kimbit, and 3 out of 59 trials for Leo). The dolphins, then, were much more likely to vary their behavior rather than repeat it when asked to change their behavior in this context $\left(\chi^{2}(2)=73.4, p<0.001\right)$. The dolphins did not differ from one another in terms of the frequency with which they began "vary" trials correctly.

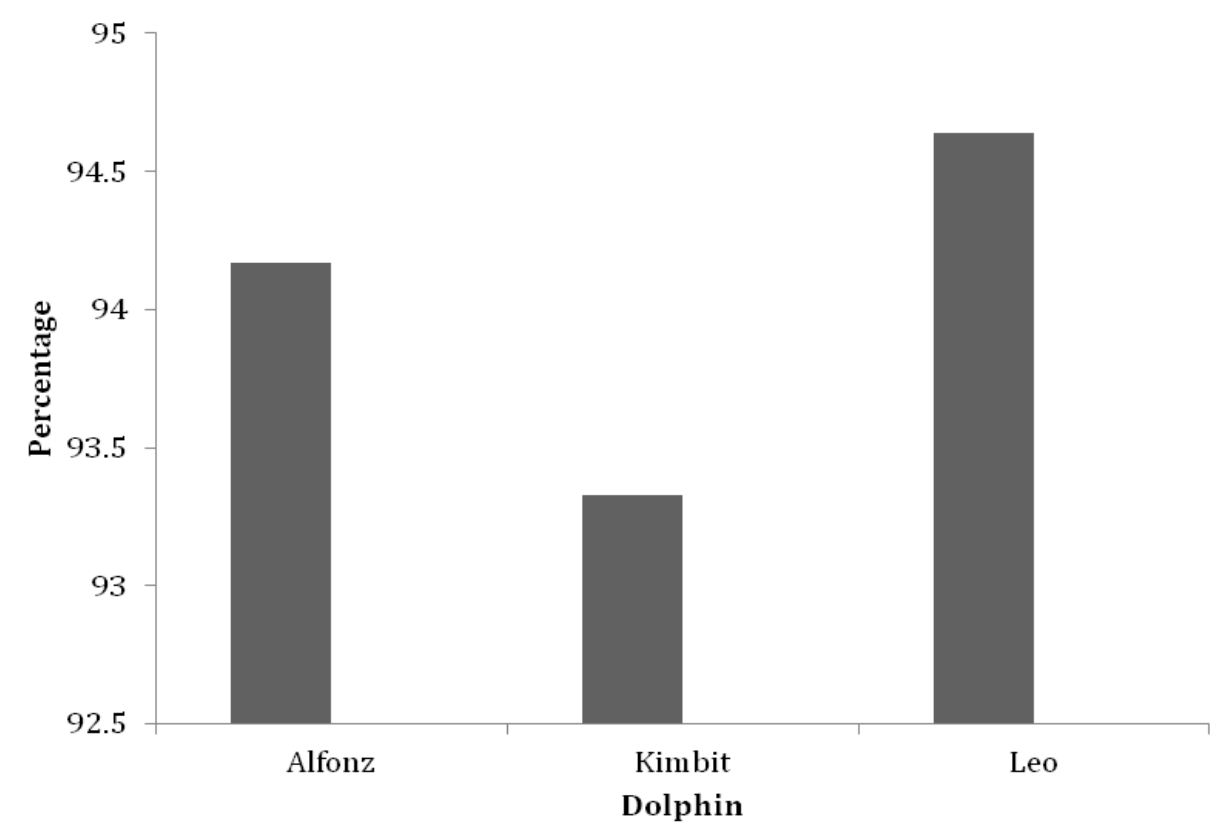

Figure 1. Percentages of correct "vary" responses for each dolphin across all test trials.

The dolphins did differ in terms of the number of behaviors that they were able to use in the "vary" trials without repeating themselves (Kruskal Wallis test, $\chi^{2}(2)=18.2, p<0.001$; see Figure 2). Post-hoc Mann-Whitney U tests revealed that the dolphins Alfonz and Leo produced significantly more behaviors during the trials than did the dolphin Kimbit (Alfonz/Kimbit $\mathrm{U}=755.5, p<0.005$; Leo/Kimbit $\mathrm{U}=679.0, p<0.001$ ). Leo also averaged more behaviors per trial than did Alfonz (9.6 for Leo, 7.2 for Alfonz). Although this difference was not statistically significant, $\mathrm{U}=1132.0, p=0.06$, it is worth noting 
that Leo's longest string of behaviors without repeating himself was much longer than Alfonz's longest string (24 for Leo, 15 for Alfonz).

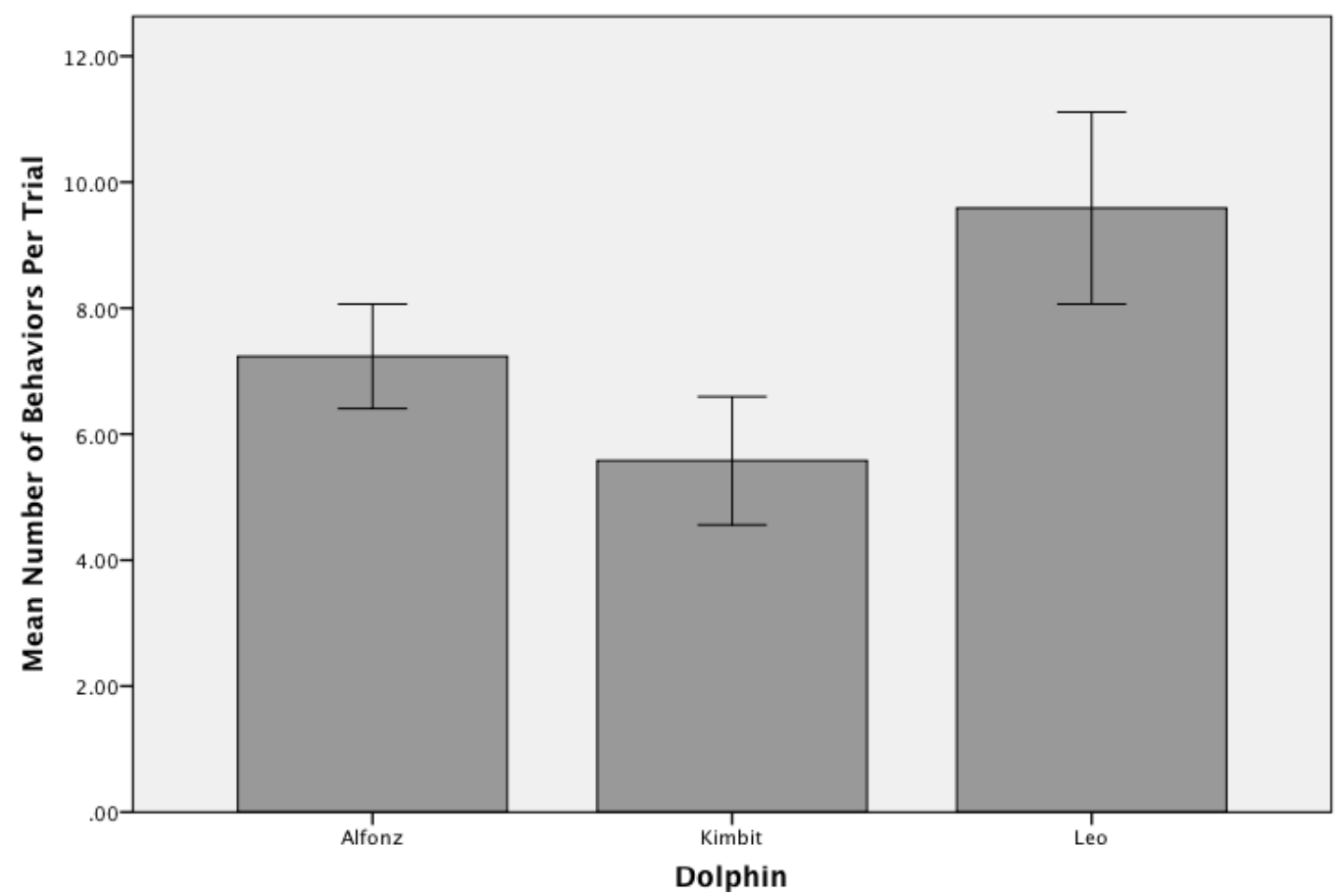

Figure 2. Mean number of different behaviors per test trial for each dolphin.

The number of components used to construct individual behaviors also varied from dolphin to dolphin (Kruskal Wallis test, $\chi^{2}(2)=27.4, p<0.001$; see Figure 3). Alfonz and Leo used significantly more components to construct the behaviors they produced than did Kimbit (Alfonz/Kimbit $\mathrm{U}=590.0, p$ $<0.001$; Leo/Kimbit $\mathrm{U}=538.5, p<0.001$ ). Alfonz and Leo did not differ significantly in terms of the average number of components they used in the behaviors they produced during test trials, $\mathrm{U}=1299.0, p$ $=0.42$. However, Leo's most complex behavior contained 10 components and Alfonz's most complex behavior contained 6 components.

We also analyzed the number of different components that were used for all of the behaviors produced by a dolphin during a "vary" trial. Recall that during a trial, the dolphin was repeatedly asked to vary its behavior (unless the dolphin repeated one of its earlier behaviors, at which time that trial was terminated). We wanted to assess the extent to which a dolphin used novel components in the behaviors he produced during successful varied behaviors rather than incorporate components he had used in earlier behaviors. As shown in Figure 4, the dolphins differed significantly in terms of the number of different components they used to vary their behaviors $\left(\chi^{2}(2)=30.5, p<0.001\right)$. Alfonz and Kimbit used similar amounts of components in their behaviors (Alfonz/Kimbit $\mathrm{U}=926.0, p<0.10$ ), but Leo averaged significantly more components per trial than did Alfonz (Alfonz/Leo $\mathrm{U}=754.0, p<0.001$ ) or Kimbit (Leo/Kimbit $\mathrm{U}=535.5, p<0.001)$. 


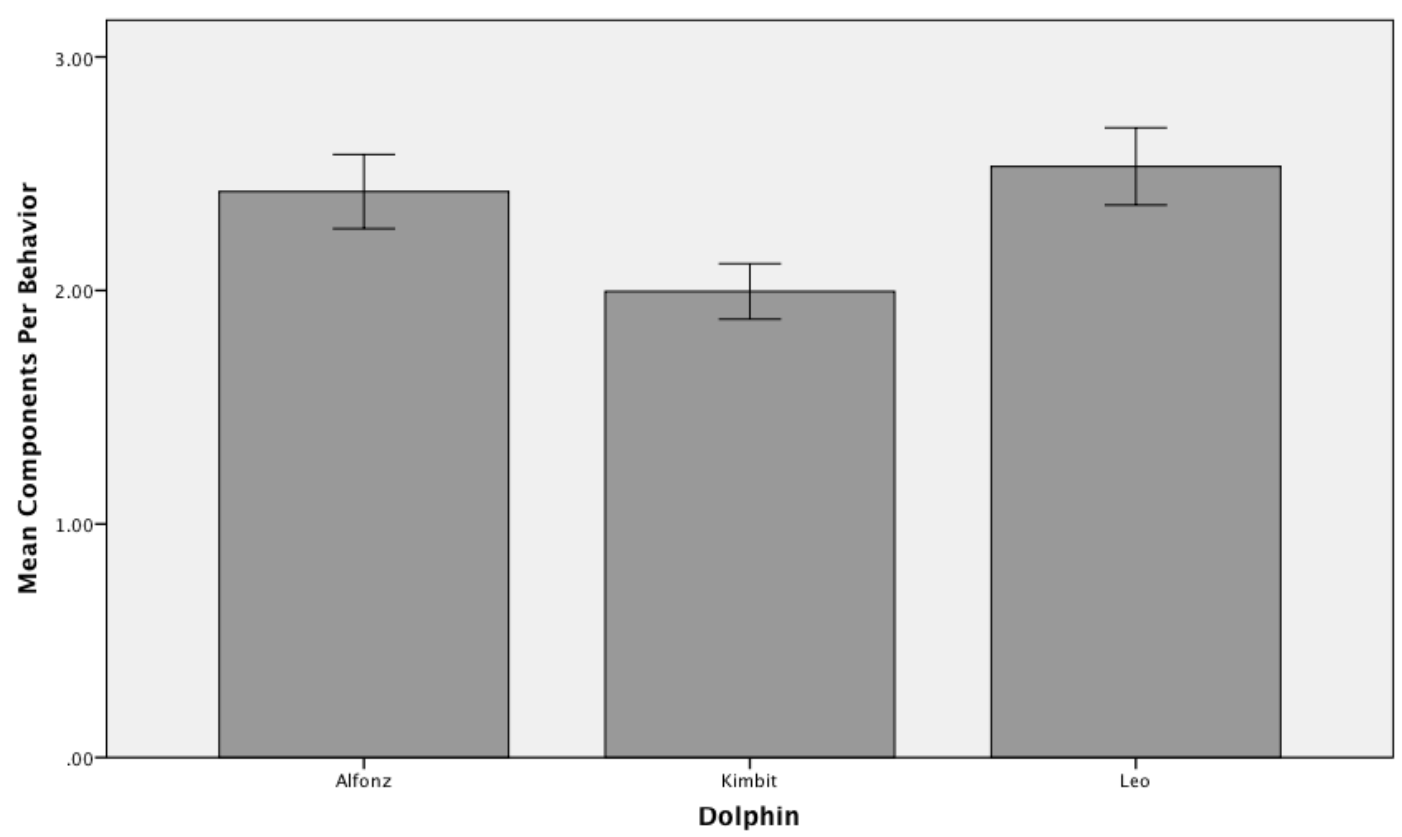

Figure 3. Mean number of different components used to "vary" each dolphin's behavior during test sessions.

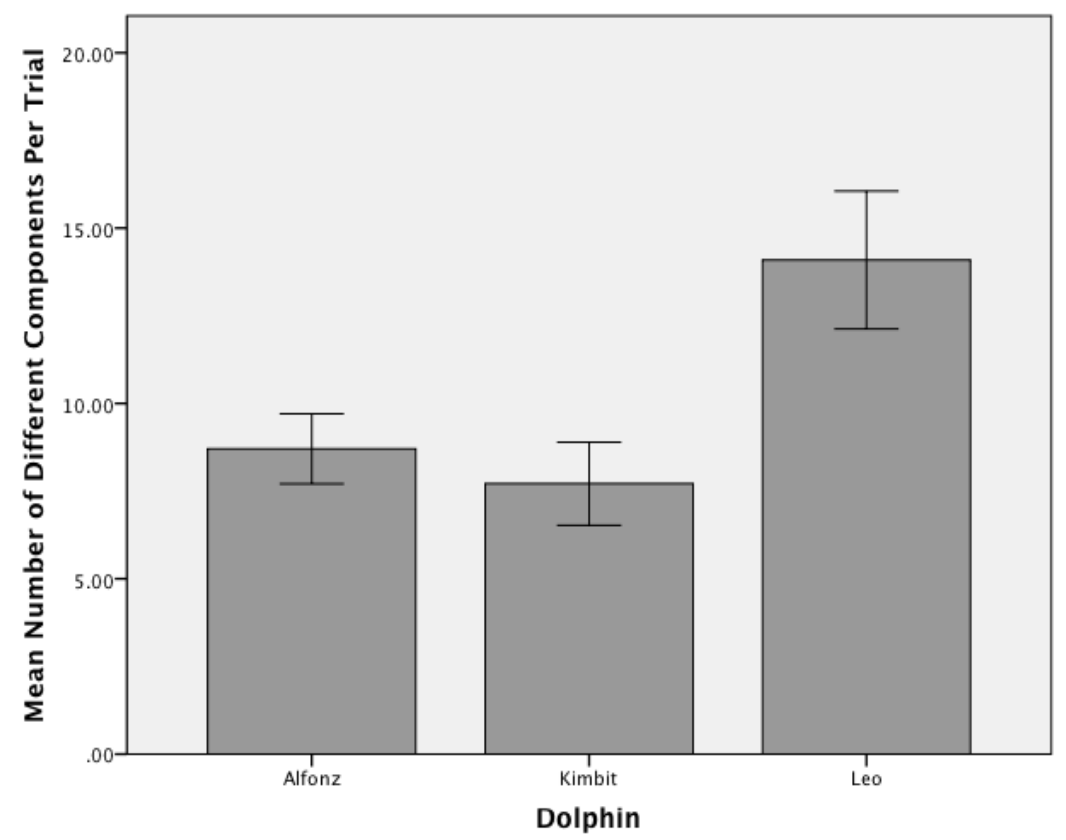

Figure 4. Mean number of different components per trial for each dolphin.

Dolphins could opt to use low, medium, or high energy behaviors to satisfy the criterion of varying their behavior (see Figure 5). We scored the first behavioral response the dolphins used to begin each trial as low (1), medium (2), or high (3) energy behaviors. Examples of low energy behaviors are vocalizing or waving a pectoral fin while otherwise remaining stationary. Medium energy behaviors 
included slowly sinking or swimming. High energy behaviors included jumping completely out of the water, lifting one's body out of the water vertically and moving forward or backward using only the fluke (tail). As shown in Figure 5, the dolphins differed significantly in terms of the energy level of the behaviors they produced $\left(\chi^{2}(2)=43.7, p<0.001\right)$. Alfonz and Kimbit did not differ from one another, with each dolphin being most likely to use low energy behaviors (Alfonz/Kimbit $\mathrm{U}=1052.8, p=0.362$. Leo was much more likely to use high energy behaviors than were Alfonz or Leo (Alfonz/Leo $\mathrm{U}=665.0$, $p<0.001$; Kimbit/Leo $\mathrm{U}=479.0, p<0.001$ ).

Given the parameters of the task the dolphins were given, it was possible for the dolphins to succeed by only producing behaviors that had some sort reinforcement history in the training context. However, as shown in Figure 6, each dolphin produced a number of behaviors that had never been reinforced by trainers prior to their occurrence in the "vary" task. In keeping with the literature on this topic, we will refer to these as "creative" behaviors (Herman, 2006; Pryor, 1975; Pryor et al., 1969). The dolphins differed significantly in terms of the rate with which creative behaviors were produced $\left(\chi^{2} 2\right)=$ 50.3, $p<0.001$ ). Kimbit produced the fewest creative behaviors (Alfonz/Kimbit $\mathrm{U}=492.0, p<0.001$; Kimbit/Leo $\mathrm{U}=311.5, p<0.001$ ), and Leo produced significantly more creative behaviors (Alfonz/Leo $\mathrm{U}=9.3, p=0.001)$.

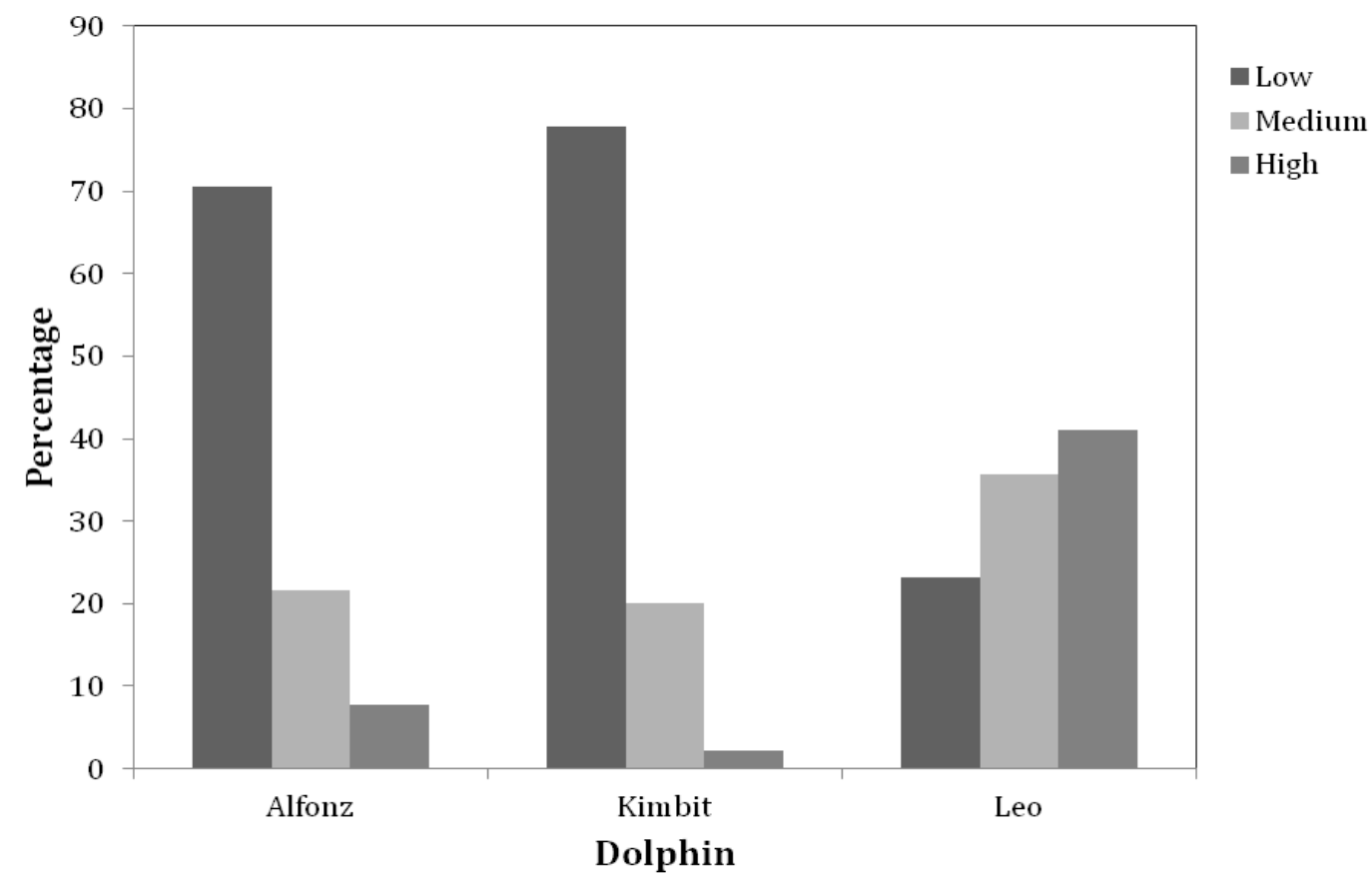

Figure 5. Percentage of low, medium, and high energy behaviors for each dolphin at the beginning of a trial. 


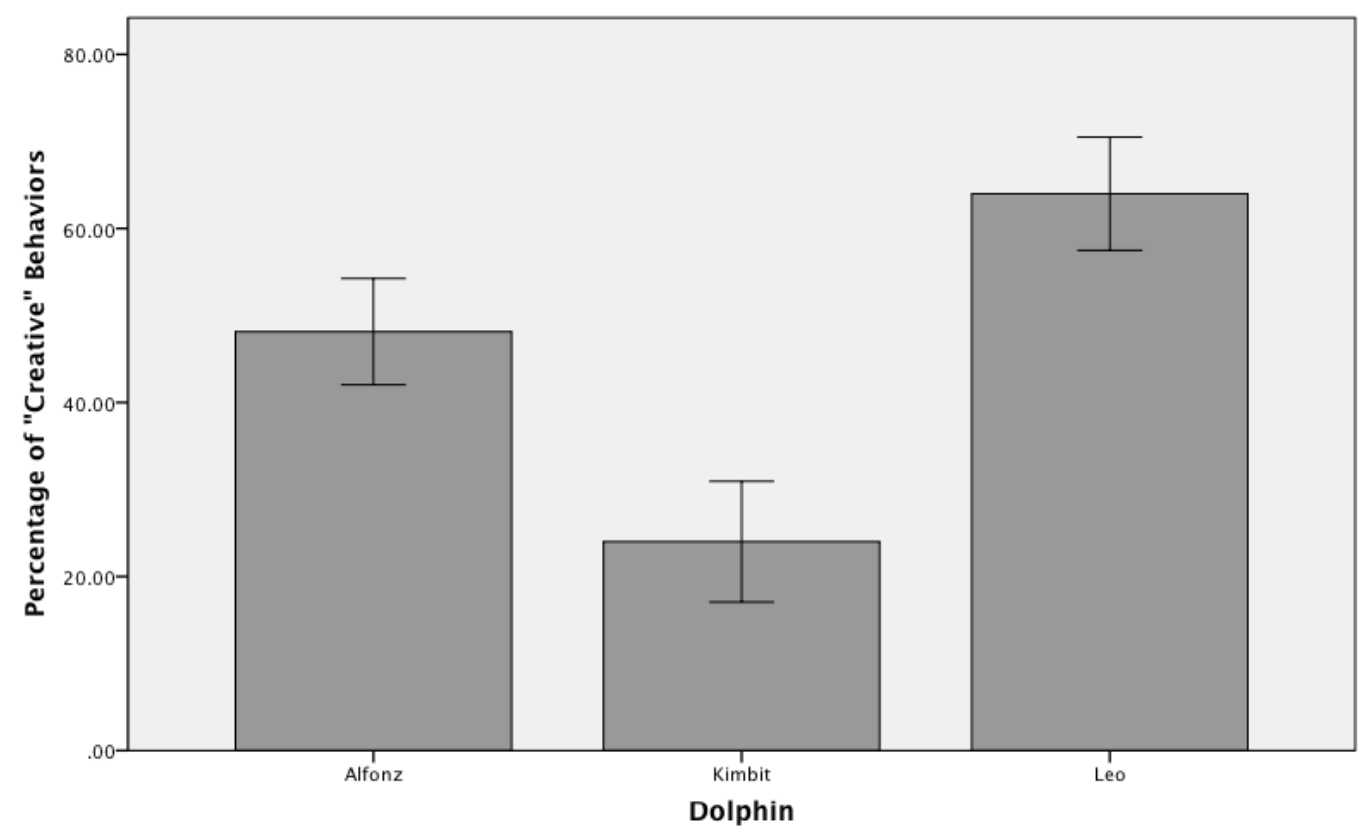

Figure 6. Mean percentage of "creative" behaviors produced by each dolphin.

Each dolphin used a variety of strategies to successfully vary their behaviors. For example, a dolphin might keep the beginning component the same but vary the components that followed it during a given trial. For example, a dolphin's first behavior consisted of a whistle followed by a pectoral wave, the next behavior was a whistle followed by a sink (the dolphin slowly sinks below the surface), and the next behavior was whistle followed by turning onto his back. Each dolphin employed this strategy approximately $20 \%$ of the time (Alfonz $=18 \%$, Kimbit $=22 \%$, and Leo $=22 \%$ ). The dolphins rarely used the opposite strategy of beginning with a different behavior and ending with the same behavior (Alfonz $=$ $3 \%$, Kimbit $=0 \%$, and Leo $=3 \%$ ).

The dolphins also differed in the use of certain strategies. For example, Alfonz and Leo both were more likely to use the strategy of "building" than was Kimbit (Alfonz $=24 \%$, Kimbit $=5 \%$, and Leo $=$ $17 \%$ ). This strategy involved the use of the preceding behavior as the basis for the next behavior, which incorporated the initial behavior and added at least one component to it. For example, a dolphin might begin by producing a sound (vocalizing), follow this by vocalizing while swimming in a circle, and follow that by vocalizing and spitting while swimming in a circle).

The opposite strategy ("deconstruction") involved using the initial behavior and then producing only a part of it in the succeeding behavior (e.g., a ventral swim with a pectoral fin wave might be followed by a ventral swim without a pectoral fin wave). Kimbit never used this strategy, and Alfonz and Leo used it occasionally (Alfonz $=6 \%$, Kimbit $=0 \%$, and Leo $=6 \%$ ).

The dolphins sometimes used the same components that had been used in the preceding behavior in the newest "vary" behavior, but varied the order in which the components occurred. Alfonz and Kimbit were four times more likely to use this strategy than was Leo (Alfonz $=9 \%$, Kimbit $=9 \%$, and Leo $=$ $2 \%)$.

The dolphins also used multiple strategies within a trial. This was especially likely when the dolphins produced long runs of varied behaviors. Alfonz and Leo were more likely to engage in multiple strategies within a trial than was Kimbit (Alfonz $=20 \%$, Kimbit $=5 \%$, Leo $=19 \%$ ).

There were times in which we were unable to ascertain any strategy that the dolphin was using during a trial. Thus, even though the dolphin was correctly varying its behavior, there was no obvious strategy being used. This occurred most often for Kimbit (Alfonz $=15 \%$, Kimbit $=47 \%$, and Leo $=14 \%$ ). 


\section{Discussion}

When dolphins are required to vary their behavior in a task such as the one used in this study, they must understand that it is not acceptable to repeat a behavior already performed and remember the behaviors they have already used. The three dolphins we studied had learned the concept to "vary" their behavior when requested to do so, and so understood that they should not repeat their behavior in this context. This is demonstrated by the fact that they rarely failed to vary their behavior at the beginning of a trial once they had achieved criterion.

The dolphins did differ in their ability to produce strings of behaviors that were not repeated, suggesting that there may have been differences in the short term memory capacity of each dolphin. Kimbit proved to be the least capable of the three, producing on average between five and six behaviors per trial. Alfonz averaged between seven and eight behaviors per trial, while Leo averaged between nine and ten behaviors per trial. The longest string of varied behaviors produced by Kimbit and Alfonz consisted of 15 behaviors, whereas Leo's maximum length was 24 behaviors.

The dolphins also used different numbers of components to build their behaviors, additional components resulting in more complex behaviors. Kimbit tended to use the fewest components per behavior (two on average, with a maximum of six). Alfonz's average use of components was slightly higher (2.4), but his most complex behavior also contained six components. Leo's average use of components was similar to that of Alfonz (2.5), but his maximum was ten.

The dolphin Kimbit that produced the shortest strings of non-repeated behaviors also used fewer components to construct his behaviors. The dolphin that produced the longest strings of non-repeated behaviors, Leo, also used the most components. Thus, Leo's ability to remember a greater number of his previously produced behaviors did not rest on his use of simpler behaviors. To the contrary, Leo produced more complex behaviors as well as longer strings of behaviors.

How did Leo do this? Not solely by reusing components that he had used previously in earlier behaviors in a trial. Leo varied his use of individual components within a trial more so than did Alfonz or Kimbit. Leo averaged 14.1 distinctive components within a trial, compared to 8.7 for Alfonz and 7.7 for Kimbit. Leo was also more likely to produce creative behaviors during the "vary" task than were Alfonz or Kimbit. Two of every three behaviors produced by Leo were creative, compared to one of two behaviors for Alfonz and one of four behaviors for Kimbit. Thus, Leo's superior performance did not depend on his use of previously reinforced behaviors or behavioral components he had used earlier in a "vary" trial. Herman (2006) reported that the dolphin in their "create" study (actually a "vary" study) produced 72 unique behaviors over the course of the study, 38 of which were creative by the definition we are using here. Herman's dolphin, like Leo and the dolphins studied by Pryor et al. (1969), seemed prone to creating new behaviors when asked to produce something different from what had just been done.

We suspect that the differences we observed among the three dolphins we studied reflect individual differences in the extent to which the dolphins embraced the challenge of not repeating themselves. All three dolphins had previously learned the task, and so rarely failed to produce at least one correct response. But they differed in their overall approach to the task. Kimbit tended to focus on low energy behaviors, and most often appeared to vary his behavior in ways that required relatively little effort. He was also the least likely of the three dolphins to produce creative behaviors in this context (although he did so approximately $25 \%$ of the time). Alfonz also focused on low energy behaviors, but was twice as likely as Kimbit to produce novel behaviors. Leo used medium or high level behaviors more often than low energy behaviors, and produced longer strings of non-repeated behaviors, more complex behaviors, more different components, and more creative behaviors than did either of the other two dolphins. At the risk of being anthropomorphic, Leo seemed the most enthusiastic of the dolphins when asked to "vary" his behavior, and his performance suggests that he relished the challenge. We know that dolphins make their play more difficult as a means to challenge themselves (Kuczaj \& Makecha, 2008; Kuczaj, Makecha, Trone, Paulos, \& Ramos, 2006; Kuczaj \& Walker, 2012; Paulos, Trone, \& Kuczaj, 2010), and it appears that Leo challenged himself in the "vary" task more so than did Alfonz or Kimbit. 
Despite the individual differences described above, it is important to remember that all three dolphins successfully solved the "vary" task. However, it is also possible that each dolphin's ability to develop strategies to facilitate their performance in the "vary" task influenced the results. Kimbit had the lowest rate of success of the three dolphins (albeit still quite successful), and he appeared to use the fewest number of clear-cut strategies and in many cases did not appear to have any discernable strategy. Alfonz and Leo used more types of strategies and were much less likely than Kimbit to produce trials without any apparent strategy. They also were more likely than Kimbit to use multiple strategies within a trial. Dolphins can switch strategies when task parameters change (Jaakkola, Guarino, Rodriguez, \& Hecksher, 2013; Kuczaj, Gory, \& Xitco, 2009), and we suspect that the dolphins in this study varied their strategies to keep the task interesting, much as dolphins modify their play to keep it interesting (Kuczaj et al., 2006; Kuczaj \& Walker, 2012).

The results of this study, combined with those demonstrating that dolphins can repeat the behavior they have just completed (Mercado et al., 1998) support the notion that dolphins represent their past actions and are able to use these representations to either repeat or modify something they have done. Although it is possible that the individual differences we observed reflect differential abilities to create and store representations of one's behavior, it is also possible that the observed differences reflect individual cognitive styles and/or the extent to which the dolphins found the "vary" game interesting rather than differences in cognitive abilities per se. Regardless, it is clear that additional research on the variability that occurs within and between dolphins when communicating, imitating, playing, and solving problems is warranted.

\section{Acknowledgements}

The authors gratefully acknowledge the logistic support of the staff and trainers at Dolphins Plus and Dolphin Cove. Their time and dedication to the training and to dolphin research was crucial to this study.

\section{References}

Benoit-Bird, K. J., \& Au, W. W. L. (2009). Cooperative prey herding by a pelagic dolphin, Stenella longirostris. Journal of the Acoustical Society of America, 125, 539-546.

Braslau-Schneck, S. (1994). Innovative behaviors and synchronization in bottlenosed dolphins (unpublished master's thesis). University of Hawaii, Honolulu, Hawaii.

Caldwell, M. C., Caldwell, D. K., \& Tyack, P. L. (1990). Review of the signature-whistle hypothesis for the Atlantic bottlenose dolphin. In S. Leatherwood \& R. Reeves (Eds.), The bottlenose dolphin (pp. 199-234). New York: Academic Press.

Daura-Jorge, F. G., Cantor, M., Ingram, S. N., Lusseau, D., \& Simões-Lopes, P. C. (2012). The structure of a bottlenose dolphin society is coupled to a unique foraging cooperation with artisanal fisherman. Biology Letters, 8, 702-705.

Duffy- Echevarria, E. E., Connor, R. C., \& St. Aubin, D. J. (2008). Observations of strand-feeding behavior by bottlenose dolphins (Tursiops truncatus) in Bull Creek, South Carolina. Marine Mammal Science, 24, 202206.

Fertl, D., \& Wilson, B. (1997). Bubble use during prey capture by a lone bottlenose dolphin (Tursiops truncatus). Aquatic Mammals, 23, 113-114.

Finn, J., Tregenza, T., \& Norman, M. (2009). Preparing the perfect cuttlefish meal: Complex prey handling by dolphins. PLoS ONE 4(1), e4217. doi:10.1371/journal.pone.0004217

Herman, L. M. (2006). Intelligence and rational behaviour in the bottlenosed dolphin. In S. Hurley \& M. Nudds (Eds.) Rational animals (pp. 439-468). New York: Oxford University Press.

Herzing, D. L. (1996). Vocalizations and associated underwater behavior of free-ranging Atlantic spotted dolphins, Stenella frontalis, and bottlenose dolphins, Tursiops truncatus. Aquatic Mammals, 22, 61-79.

Highfill, L., \& Kuczaj, S. A., II (2007). Do bottlenose dolphins (Tursiops truncatus) have distinct and stable personalities? Aquatic Mammals, 33, 380-389.

Jaakkola, K., Guarino, E., Rodriguez, M., \& Hecksher, J. (2013). Switching strategies: A dolphin's use of passive and active acoustics to imitate motor actions. Animal Cognition, 16, 701-709. 
Janik, V. M., \& Sayigh, L. S. (2013). Communication in bottlenose dolphins: 50 years of signature whistle research. Journal of Comparative Physiology, 199, 479-489.

Krützen, M., Mann, J., Heithaus, M. R., Connor, R. C., Bejder, L., \& Sherwin, W. B. (2005). Cultural transmission of tool use in bottlenose dolphins. Proceedings of the National Academy of Sciences of the United States of America, 102, 8939-8943.

Kuczaj, S. A., II (in press). Language learning in cetaceans. In P. Brooks \& V. Kempe (Eds.), Encyclopedia of language development. New York: Sage.

Kuczaj, S. A., II, Gory, J. D., \& Xitco, M. J., Jr. (2009). How intelligent are dolphins? A partial answer based on their ability to plan their behavior when confronted with novel problems. Japanese Journal of Animal Psychology, 59, 99-115.

Kuczaj, S. A., II, Highfill, L., \& Byerly, H. (2012). The importance of considering context in the assessment of personality characteristics: Evidence from ratings of dolphin personality. International Journal of Comparative Psychology, 25, 309-329.

Kuczaj, S. A., II, \& Makecha, R. (2008). The role of play in the evolution and ontogeny of contextually flexible communication. In U. Griebel \& K. Oller (Eds.), Evolution of communicative flexibility: Complexity, creativity, and adaptability in human and animal communication (pp. 253-277). Cambridge, MA: MIT Press.

Kuczaj, S. A., II, Makecha, R. N., Trone, M., Paulos, R. D., \& Ramos, J. A. (2006). The role of peers in cultural transmission and cultural innovation: Evidence from dolphin calves. International Journal of Comparative Psychology, 19, 223-240.

Kuczaj, S. A., II, \& Walker, R. T. (2012). Dolphin problem solving. In T. Zentall \& E. Wasserman (Eds.), Handbook of comparative cognition. Oxford, UK: Oxford University Press.

Kuczaj, S. A., II, Yeater, D., \& Highfill, L. (2012). How selective is social learning in dolphins? International Journal of Comparative Psychology, 25, 221-236.

Lewis, J. S., \& Schroeder, W. W. (2003). Mud plume feeding: A unique foraging behavior of the bottlenose dolphin in the Florida Keys. Gulf of Mexico Science, 21, 92-97.

Mann, J., Sargeant, B. L., Watson-Capps, J. J., Gibson, Q. A., Heithaus, M. R., Connor, R. C., \& Patterson, E. (2008). Why do dolphins carry sponges? PLOS ONE 3(12): e3868. doi:10.1371/journal.pone.0003868

Mercado, E., Murray, S. O., Uyeyama, R. K., Pack, A. A., \& Herman, L. M. (1998). Memory for recent actions in the bottlenosed dolphin (Tursiops truncatus): Repetition of arbitrary behaviors using an abstract rule. Animal Learning \& Behavior, 26, 210-218.

Nowacek, D. P. (2002). Sequential foraging behavior of bottlenose dolphins, Tursiops truncatus, in Sarasota Bay, FL. Behaviour, 139, 1125-1145.

Paulos R. D., Trone, M., \& Kuczaj, S. A., II (2010). Play in wild and captive cetaceans. International Journal of Comparative Psychology, 23, 701-722.

Peddemors, V. M., \& Thompson, G. (1994). Beaching behavior during shallow water feeding by humpback dolphins, Sousa plumbea. Aquatic Mammals, 21, 65-67.

Pryor, K. (1975). Lads before the wind. New York: Harper \& Row.

Pryor, K., Haag, R., \& O'Reilly, J. (1969). The creative porpoise: Training for the novel behavior. Journal of the Experimental Analysis of Behavior, 121, 653-661.

Rossbach, K. A., \& Herzing, D. L. (1998). Underwater observations of benthic-feeding dolphins (Tursiops truncatus) near Grand Bahama. Marine Mammal Science, 13, 498-504.

Sargeant, B. L., Mann, J., Berggren, P., \& Krützen, M. (2005). Specialization and development of beach hunting, a rare foraging behavior, by bottlenose dolphins (Tursiops sp.). Canadian Journal of Zoology, 83, 14001410.

Smolker, R., Richards, A., Connor, R., Mann, J., \& Berggren, P. (1997). Sponge carrying by dolphins (Delphinidae, Tursiops sp.): A foraging specialization involving tool use? Ethology, 103, 454-465.

Spitz, J., Richard, E., Meynier, L., Pusineri, C., \& Ridoux, V. (2006). Dietary plasticity of the oceanic striped dolphin, Stenella coeruleoalba, in the neritic waters of the Bay of Biscay. Journal of Sea Research, 55, 309-320.

Tyack, P. L., \& Sayigh L. S. (1997). Vocal learning in cetaceans. In C. Snowdon \& M. Hausberger (Eds.), Social influences on vocal development (pp. 208-233). Cambridge, UK: Cambridge University Press. 